\title{
Three-dimensional positioning study of the internal structure of petrous bone with the external lower lip of internal acoustic pore as anatomical landmark
}

\author{
Xian-Feng Wei ${ }^{1,2}$, Peng $\mathrm{Lin}^{1}, \mathrm{Li} \mathrm{Li}^{1}$ and Yun-Sheng $\mathrm{Li}^{2}$ \\ ${ }^{1}$ Department of Otolaryngology Head and Neck, Tianjin First Central Hospital, Tianjin, China \\ ${ }^{2}$ Department of Anatomy and histology \& embryology, Basic Medical College, Tianjin Medical University, Tianjin, China
}

\begin{abstract}
Objective: To conduct exact positioning study of the structures inside the petrous bone by creating three dimensional coordinate system with the external lower lip of the internal acoustic pore as its origin.

Research design: The three-dimensional model of the petrous bone was established by making nonstaining celloidin section from the right petrous bone of a dead man in an attempt to create original three dimensional coordinate which was later transformed to physical coordinate system. The three dimensional coordinate of each structure inside and outside the petrous bone under the physical coordinate system was measured by choosing the external lower lip of the internal acoustic pore as origin.

Results: The three-dimensional model of structures inside and outside the petrous bone was established and coordinate values of each structure inside and outside the petrous bone under the physical coordinate system and relevant distances were measured by selecting the external lower lip of the internal acoustic pore as origin.

Conclusion: A certain surface mark of the petrous bone can be chosen as the coordinate origin to make exact positioning study of the structures inside and outside the petrous bone.
\end{abstract}

\section{Introduction}

Transpetrosal approach is the common operative route of treating diseased regions like the interior of petrous bone, the ventral aspect of brain stem, trans-middle and trans-posterior cranial fossa. Preoperative familiarity with the anatomical structures related to petrous bone and the quantification of data information are among important prerequisites and determinants which have an impact on surgical results. We established a three dimensional coordinate system during the experiment using the external lower lip of internal acoustic pore as origin to conduct an exact positioning research into the internal composition of the petrous bone.

\section{Materials and methods}

Three dimensional modeling of anatomic structures of the petrous bone

The cadaver comes from department of Anatomy and histology \& embryology, Tianjin Medical University. This research project did not require approval by the institutional review committee because the cadaver had previously been donated for research and the protocols already signed. The Ethics Committee of Tianjin Medical University approved the experimental protocol.

The right petrous bone comes from a 75-year-old man with no history of otological disease. The specimens were fixed in formalin, decalcified in chlorhydric acid, embedded in celloidin. Hardened in ethanol, the celloidin block were serially sectioned in the horizontal plane at a thickness of $40 \mu \mathrm{m}$ and 487 successive celloidin sections of the petrous bone without staining were finally obtained. The highest resolution and best definition of the digital camera were used to generate images which were saved in the digital JPEG picture format. The contours of all structures in petrous bone were manually precisely outlined and reconstructed with Amira software, and threedimensional data set of temporal bone was obtained [1].

\section{Creation of original coordinate system}

After opening Amira software, the data of image slices were introduced into the file named data in Amira software and the icon of the image file would appear in Pool. Then we clicked the right-hand button of the icon module and chose Isosurface module in Display order, thus displaying the three-dimensional model of the petrous bone. By clicking the right-hand button of the file icon and then selecting Local Axis module in Display order, the original coordinate system would be seen in the three dimensional display windows.

\section{Creation of the physiological coordinate system}

Rotating the coordinate system to be in the same plane as the lateral semicircular canal: After the three-dimensional model of the

Correspondence to: Yun-Sheng Li, Department of Anatomy and histology and embryology, Basic Medical College, Tianjin Medical University, QiXiangTai Road 22, HePing District, Tianjin, 300070, China, Tel. +862283336818; E-mail: submission2014@163.com,weixianfeng3@163.com

Key words: the internal acoustic pore, three dimensional coordinate system, physical coordinate system, positioning study

Received: April 02, 2016; Accepted: April 26, 2016; Published: April 29, 2016 
Wei XF (2016) Three-dimensional positioning study of the internal structure of petrous bone with the external lower lip of internal acoustic pore as anatomical landmark

lateral semicircular canal was obtained, the $\mathrm{x}$-y axis was parallel to the plane of the lateral semicircular canal by turning the ball around the coordinate system of the petrous bone. In the Properties zone of the data icon of the petrous bone, the rotation angle of the $3 \mathrm{D}$ coordinate system could be seen by clicking Dialoge box in the parameter option of Manipulator: $38.44(-0.65,0.80,0.04)$ (Figure 1).

Turning the coordinate system in accordance with the long axis of the petrous bone: The included angle between the long axis of the petrous bone on the right side and the connecting line of both ears measured on the head of dead body used in the experiment was $37.2^{\circ}$. As the included angle measured in the above coordinate system was $25^{\circ}$ after again resurfacing the petrous bone, we rotated the $3 \mathrm{D}$ coordinate along the $\mathrm{Z}$ axis by $12.2^{\circ}$ and then the angle reached $37.2^{\circ}$.

Turning the coordinate system to physiological coordinate: Physiologically, the lateral semicircular canals on both sides situate in the same plane, while being parallel to the ground when leaning head forward by $30^{\circ}$. Therefore, through turning the above coordinate along the $\mathrm{X}$ axis by $30^{\circ}$, the $3 \mathrm{D}$ coordinate system was similar to that in the physiological condition (i.e. under the condition of anatomical position), with the $\mathrm{x}$ axis (i.e. frontal axis) being red pointing outward, the $\mathrm{y}$ axis (i.e. sagittal axis) green pointing forward and $\mathrm{z}$ axis (i.e. vertical axis) blue pointing above. Under this circumstance, the total rotation angle of the coordinate system was $61.55(-0.81,0.46,0.35)$, that is $\left(-50.02^{\circ}, 28.51^{\circ}\right.$ and $\left.21.77^{\circ}\right)$. The spatial position under the original and physiological coordinates of the petrous bone and its surface structures are shown in Figure 2.
Measurement and calculation of the 3D coordinate of the exterior and interior structures of the petrous bone under physiological coordinate with the external lower lip of the internal acoustic pore as its origin

Measurement of the 3D coordinates of structures under original coordinate: We made $3 \mathrm{D}$ reconstruction by drawing contour line of the external lower lip of the internal acoustic pore to establish its original coordinate. Through clicking Transform Editor in the Properties zone of module .surf* and then clicking Interact in the display window on the right, the origin of the $3 \mathrm{D}$ coordinate could be dragged to anywhere. By clicking the Dialogue box in the parameter option of Manipulator in the Properties zone, the 3D coordinate of positions where the coordinate origin reached would be observed. As for the coordinate of the nearest and the farthest distances between each structure point and the coordinate origin, the distances could be measured first and then the coordinate.

Conversion of original coordinates to physiological coordinate: The original 3D coordinate of the interior structures of the petrous bone was measured by using the external lower lip of the internal acoustic pore as its coordinate origin. After selecting the interface of Relative Local in dialog box of Transform Editor, we filled in the option of Rotate with $-61.55(-0.81,0.46,0.35)$ and in the option of Translation with the original $3 \mathrm{D}$ coordinate (Figure 3 , original coordinate of the hindmost crus osseum simplex) respectively before clicking Apply. Then by entering the interface of Absolute, the coordinate shown in the option of Translation was the physiological coordinate (Figure 4,

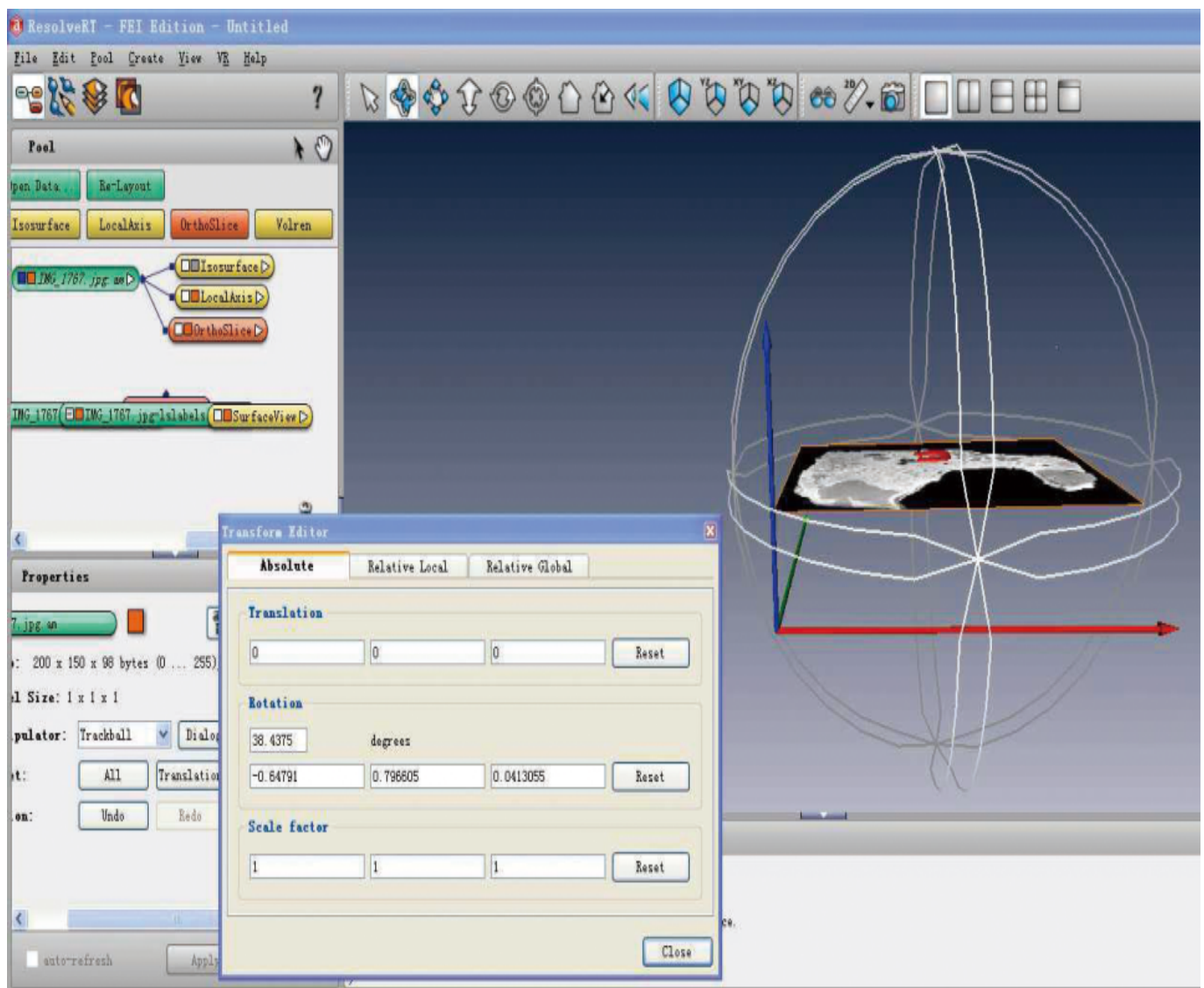

Figure 1. Rotating the coordinate system to be in the same plane as the lateral semicircular canal. 
Wei XF (2016) Three-dimensional positioning study of the internal structure of petrous bone with the external lower lip of internal acoustic pore as anatomical landmark

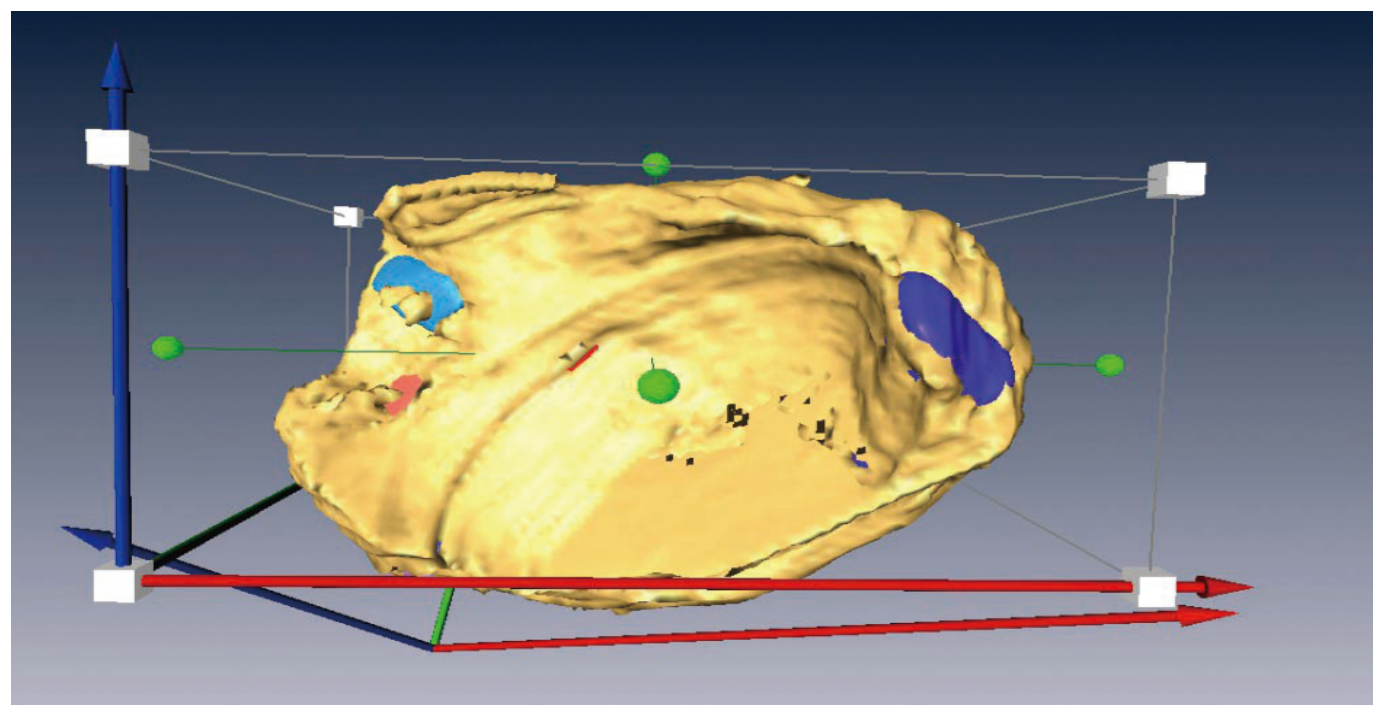

Figure 2. The spatial position of the petrous bone and its surface structures under the original and physiological coordinates, with the coordinate axis in the form of white cubes representing the physiological coordinate.

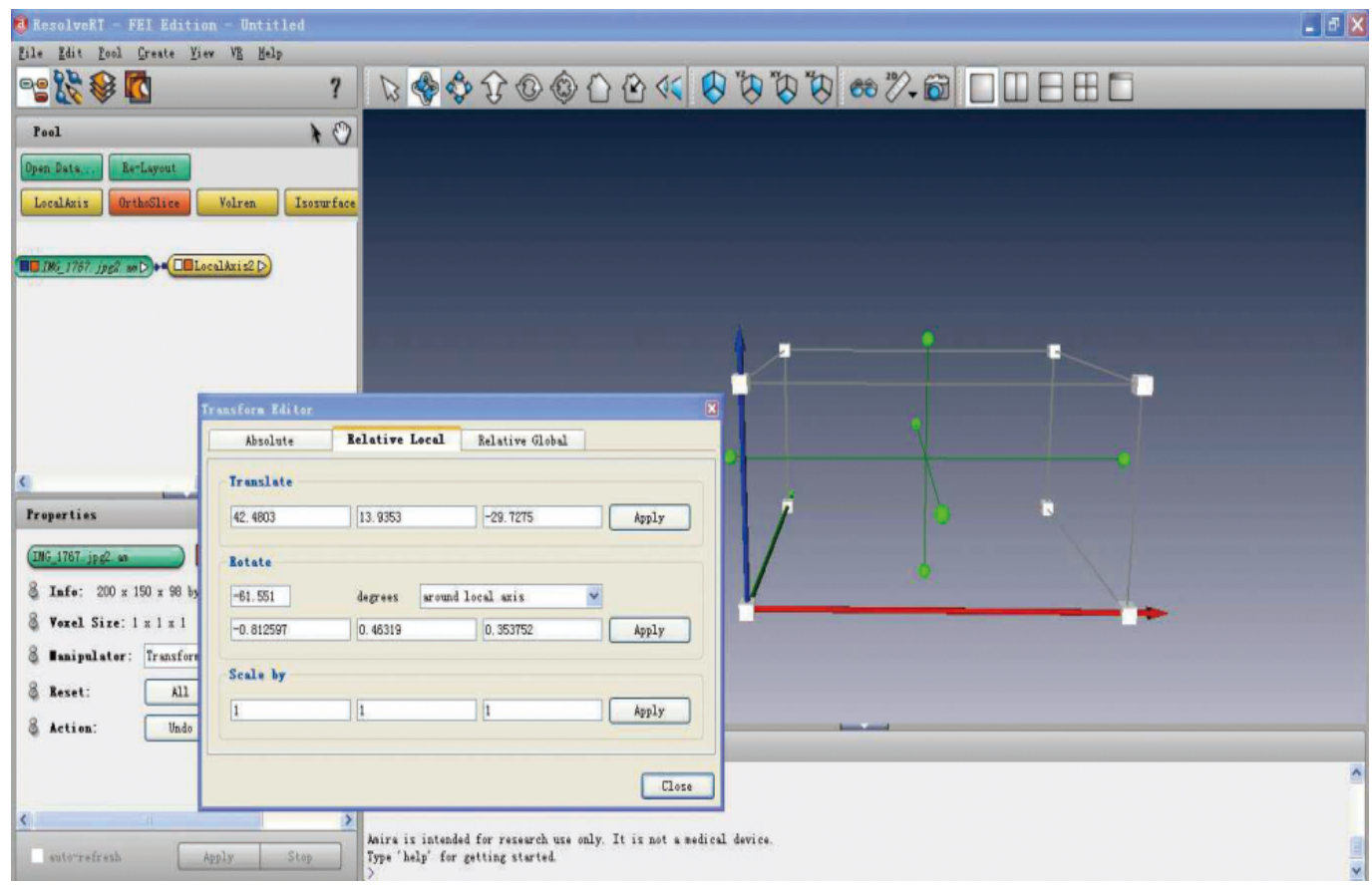

Figure 3. Original coordinate of the hindmost crus osseum simplex shown in the option of Translate

physiological coordinate of the hindmost crus osseum simplex).

Conversion of physiological coordinate to its coordinate values: The length $(\mathrm{mm})$ of each pixel could be figured out to be $0.17 \mathrm{~mm}$ through measuring the pixel of the corresponding structure by utilizing measuring tools. The coordinate unit applied to the above-mentioned measurement and calculation was pixel and the physiological coordinate was converted to coordinate values $(\mathrm{mm})$ based on the corresponding relation between pixel and length.

\section{Results}

The physiological coordinate values measured from the exterior and interior compositions of the petrous bone using the external lower lip of the internal acoustic pore as its origin are shown in Table 1 to 6. On the basis of $3 \mathrm{D}$ physiological coordinate values, the distance between each coordinate point and the original point was calculated according to the formula $\sqrt{\mathrm{X}^{2}+Y^{2}+Z^{2}}$. The nearest and the farthest distances between each structure point and the coordinate origin could be calculated from 3D physiological coordinate values in accordance with the formula. Also, the distances could be measured in the original coordinate and then converted to length (values inside the brackets of the tables). By testing the results of the two methods with paired T test, no statistical difference was observed $(P=0.809)$.

\section{Discussion}

Positioning was initially conducted primarily through measuring 
Wei XF (2016) Three-dimensional positioning study of the internal structure of petrous bone with the external lower lip of internal acoustic pore as anatomical landmark

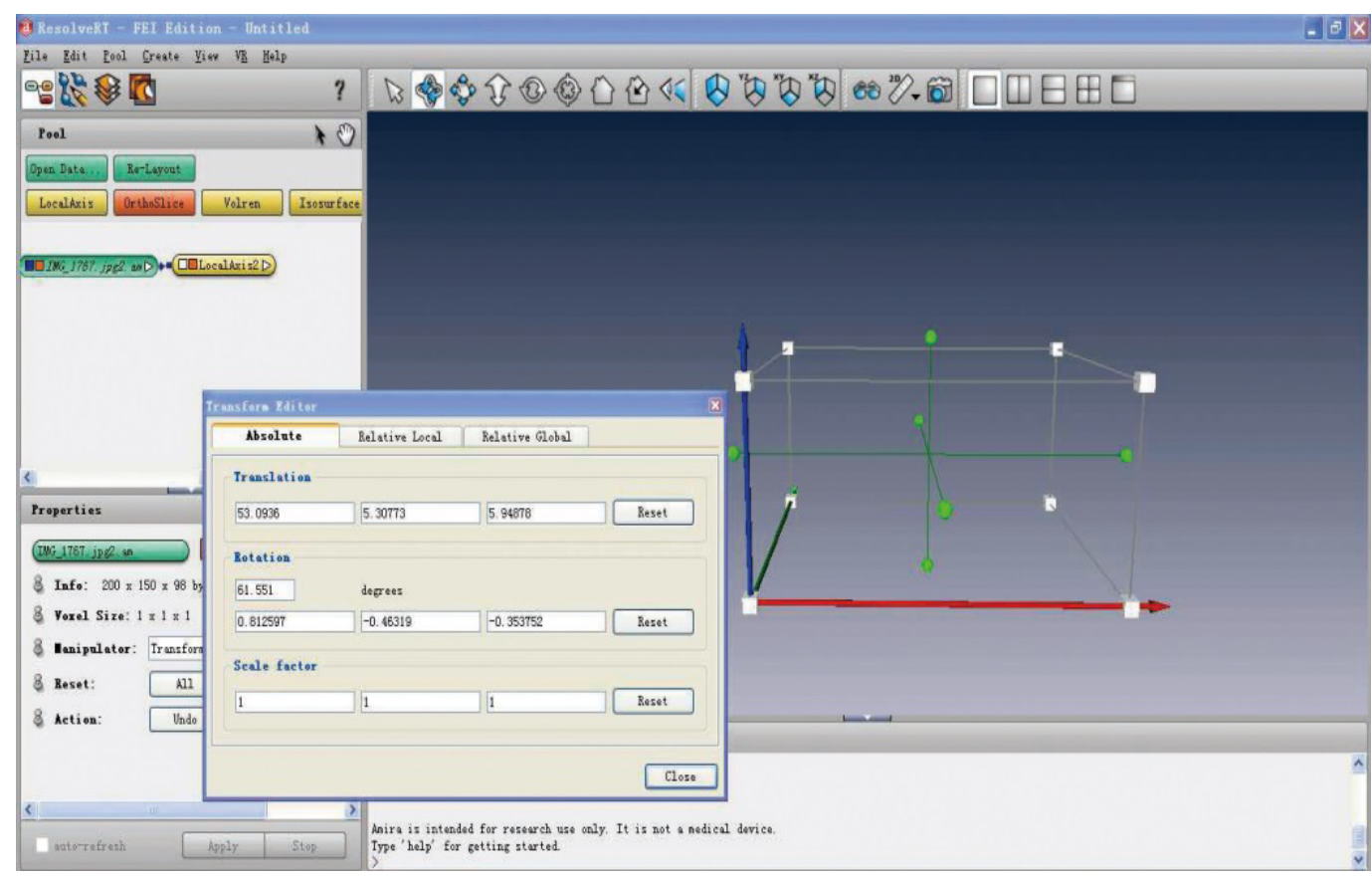

Figure 4. Physiological coordinates of the hindmost crus osseum simplex shown in the option of Translation.

Table 1. The physiological coordinate values measured from the exterior and interior compositions of the petrous bone using the external lower lip of the internal acoustic pore as its origin $(\mathrm{mm})$.

\begin{tabular}{|c|c|c|c|c|c|c|}
\hline & & & $\mathbf{X}$ axis & Y axis & $\mathrm{Z}$ axis & distance \\
\hline \multirow{12}{*}{ 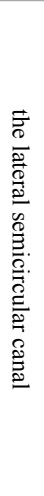 } & \multirow{12}{*}{ 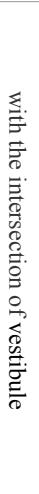 } & the backmost of the crus simplex & 9.034892 & 0.903214 & 1.012299 & 9.136182 \\
\hline & & the foremost of the crus simplex & 9.229889 & 1.275921 & 1.051126 & 9.376763 \\
\hline & & the uppermost of the crus simplex & 9.138134 & 1.201052 & 1.217106 & 9.29674 \\
\hline & & the lowest of the crus simplex & 9.070117 & 1.109802 & 0.712221 & 9.165476 \\
\hline & & the backmost of the ampulla & 9.954827 & 2.841042 & 2.03781 & 10.55096 \\
\hline & & the foremost of the ampulla & 9.54831 & 3.834285 & 1.865683 & 10.45719 \\
\hline & & the uppermost of the ampulla & 9.51106 & 3.593547 & 2.503052 & 10.47087 \\
\hline & & the lowest of the ampulla & 9.739359 & 3.357692 & 1.501327 & 10.41072 \\
\hline & & the exterior of topmost semicircular canal & 13.44529 & 1.598853 & 1.294245 & 13.60174 \\
\hline & & the interior of topmost semicircular canal & 12.41154 & 2.030271 & 1.4782 & 12.66307 \\
\hline & & the nearest point & 9.015578 & 0.911111 & 0.838452 & $\begin{array}{c}9.100207 \\
(9.109154)\end{array}$ \\
\hline & & the farthest point & 13.42578 & 2.195897 & 1.464127 & $\begin{array}{c}13.68273 \\
(13.65267)\end{array}$ \\
\hline
\end{tabular}

Table 2. The physiological coordinate values measured from the exterior and interior compositions of the petrous bone using the external lower lip of the internal acoustic pore as its origin $(\mathrm{mm})$.

\begin{tabular}{|c|c|c|c|c|c|c|}
\hline & & & $\mathrm{X}$ axis & $\mathrm{Y}$ axis & $\mathrm{Z}$ axis & distance \\
\hline \multirow{12}{*}{ 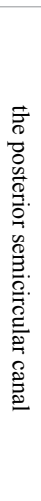 } & \multirow{12}{*}{ 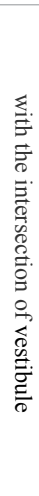 } & the backmost of the crus simplex & 8.010764 & 0.76208 & 1.164243 & 8.130718 \\
\hline & & the foremost of the crus simplex & 7.648916 & 1.376913 & 1.517189 & 7.918565 \\
\hline & & the uppermost of the crus simplex & 7.463585 & 1.249059 & 1.197383 & 7.661526 \\
\hline & & the lowest of the crus simplex & 8.200366 & 0.864032 & 1.497179 & 8.380579 \\
\hline & & the backmost of the ampulla & 8.223271 & 1.294223 & 0.414069 & 8.334786 \\
\hline & & the foremost of the ampulla & 8.070935 & 1.898458 & 0.255123 & 8.295132 \\
\hline & & the uppermost of the ampulla & 7.650992 & 1.74859 & 0.349573 & 7.856045 \\
\hline & & the lowest of the ampulla & 8.462784 & 1.564836 & 0.309408 & 8.611803 \\
\hline & & the exterior of topmost semicircular canal & 11.64733 & -2.30137 & 0.693666 & 11.89276 \\
\hline & & the interior of topmost semicircular canal & 11.03761 & -1.86812 & 0.772553 & 11.22121 \\
\hline & & the nearest point & 7.380185 & 0.855083 & 1.424343 & $\begin{array}{c}7.564856 \\
(7.574229)\end{array}$ \\
\hline & & the farthest point & 11.97332 & -1.75398 & -0.18901 & $\begin{array}{c}12.10258 \\
(12.10073)\end{array}$ \\
\hline
\end{tabular}


Wei XF (2016) Three-dimensional positioning study of the internal structure of petrous bone with the external lower lip of internal acoustic pore as anatomical landmark

Table 3. The physiological coordinate values measured from the exterior and interior compositions of the petrous bone using the external lower lip of the internal acoustic pore as its origin (mm).

\begin{tabular}{|c|c|c|c|c|c|c|}
\hline & & & $\mathbf{X}$ axis & Y axis & $\mathrm{Z}$ axis & distance \\
\hline \multirow{8}{*}{ 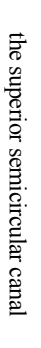 } & \multirow{8}{*}{ 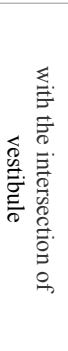 } & the backmost of the ampulla & 9.369394 & 2.537766 & 2.522349 & 10.02936 \\
\hline & & the foremost of the ampulla & 9.309035 & 3.637893 & 2.638524 & 10.33703 \\
\hline & & the innermost of the ampulla & 8.999957 & 2.978675 & 2.824604 & 9.891922 \\
\hline & & the outermost of the ampulla & 9.856741 & 2.991148 & 2.331164 & 10.56109 \\
\hline & & the exterior of topmost semicircular canal & 8.927941 & -0.25883 & 6.093161 & 10.81211 \\
\hline & & the interior of topmost semicircular canal & 9.091406 & 0.069613 & 5.643864 & 10.70101 \\
\hline & & the nearest point & 7.369753 & 0.882747 & 1.513968 & $\begin{array}{c}7.575262 \\
(7.574229)\end{array}$ \\
\hline & & the farthest point & 10.75898 & 2.49747 & 5.253989 & $\begin{array}{c}12.231 \\
(12.23516)\end{array}$ \\
\hline
\end{tabular}

Table 4. The physiological coordinate values measured from the exterior and interior compositions of the petrous bone using the external lower lip of the internal acoustic pore as its origin (mm).

\begin{tabular}{|c|c|c|c|c|c|}
\hline & & $\mathrm{X}$ axis & $\mathrm{Y}$ axis & $\mathrm{Z}$ axis & distance \\
\hline \multirow{7}{*}{$\begin{array}{l}\vec{F} \\
0 \\
8 \\
\delta \\
\frac{2}{8} \\
\bar{D}\end{array}$} & the backmost of the origin of the basal turn & 8.674423 & 3.548673 & -0.24298 & 9.375379 \\
\hline & the foremost of the origin of the basal turn & 8.309564 & 4.525955 & 0.552813 & 9.478329 \\
\hline & the innermost of the origin of the basal turn & 7.89915 & 4.157436 & 0.095107 & 8.926919 \\
\hline & the outermost of the origin of the basal turn & 9.014761 & 3.772939 & 0.306408 & 9.777263 \\
\hline & the apex & 7.657611 & 6.81153 & 1.228337 & 10.32205 \\
\hline & the nearest point & 4.104395 & 5.34617 & 1.502401 & $\begin{array}{c}6.905418 \\
(6.915674) \\
\end{array}$ \\
\hline & the farthest point & 7.802647 & 6.787537 & 1.34977 & $\begin{array}{c}10.42947 \\
(10.43307)\end{array}$ \\
\hline \multirow{2}{*}{ 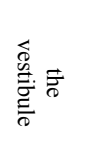 } & the nearest point & 7.29105 & 1.432128 & 0.914127 & $\begin{array}{c}7.48639 \\
(7.487442)\end{array}$ \\
\hline & the farthest point & 9.856469 & 3.058212 & 2.316819 & $\begin{array}{c}10.57688 \\
(10.55729)\end{array}$ \\
\hline \multirow{4}{*}{ 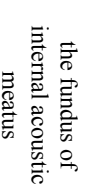 } & the posterior transverse ridge & 6.086814 & 2.48595 & 2.298577 & 6.965106 \\
\hline & the anterior transverse ridge & 5.380936 & 3.69963 & 2.998091 & 7.185422 \\
\hline & the superior border & 6.413675 & 3.237213 & 3.594296 & 8.033289 \\
\hline & the inferior border & 3.802021 & 3.177977 & 0.838049 & 5.025657 \\
\hline \multirow{6}{*}{ 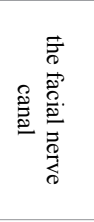 } & the center of the origin & 6.031305 & 3.267843 & 3.377075 & 7.645919 \\
\hline & the starting point of greater superficial petrosal nerve & 7.51438 & 6.51319 & 3.278462 & 10.47071 \\
\hline & the uppermost of geniculate ganglion & 7.997865 & 5.563187 & 4.013541 & 10.53676 \\
\hline & the foremost of geniculate ganglion & 8.927788 & 6.795075 & 2.995317 & 11.61251 \\
\hline & the backmost of geniculate ganglion & 9.412549 & 5.232871 & 3.021489 & 11.18519 \\
\hline & the plane of pyramidal eminence & 13.03242 & 2.4738 & -0.1292 & 13.26576 \\
\hline \multirow{4}{*}{ 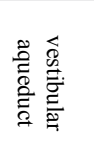 } & the starting point & 7.622267 & 1.489749 & 1.572926 & 7.924166 \\
\hline & turning & 7.001235 & 0.159407 & 2.18926 & 7.337272 \\
\hline & the external lower port & 6.479122 & -2.78227 & -1.70491 & 7.254431 \\
\hline & the external upper port & 7.732826 & -2.74537 & -0.31784 & 8.211862 \\
\hline
\end{tabular}

structures and data related to petrous bone during operation or of the head of dead body and the methods of operative approach and lesion removal were thus proposed on the basis of fissures, pores as well as the interrelations between these structures. In recent years, great development has been made in the study of temporal bone anatomy using the technology of computed $3 \mathrm{D}$ reconstruction.

\section{Creation of the physiological coordinate system}

It makes sense to study under physiological coordinate system when positioning the internal structures in accordance with the surface markers of the petrous bone. Physiological coordinate system refers to the $3 \mathrm{D}$ coordinate created under the condition of anatomical position. The 3D coordinate (original) in Amira software is automatically constructed according to the planar system of image slices rather than physiological condition, so the original coordinate needs to be turned into physiological coordinate. According to the fact that the lateral semicircular canals on both sides situate in the same plane under physiological condition while being parallel to the ground when leaning head forward by $30^{\circ}$, the original coordinate is then rotated to become physiological coordinate in accordance with the physiological included angle between the long axis of the petrous bone and the frontal axis. As the software is unable to make direct coordinate measurement in physiological coordinate, the original coordinate of relevant structures need to be measured in physiological coordinate of the petrous bone using each mark point as original point and then converted to physiological coordinate. 
Wei XF (2016) Three-dimensional positioning study of the internal structure of petrous bone with the external lower lip of internal acoustic pore as anatomical landmark

Table 5. The physiological coordinate values measured from the exterior and interior compositions of the petrous bone using the external lower lip of the internal acoustic pore as its origin (mm)

\begin{tabular}{|c|c|c|c|c|c|c|c|}
\hline & & & & $\mathrm{X}$ axis & Y axis & $\mathrm{Z}$ axis & distance \\
\hline \multirow{5}{*}{ 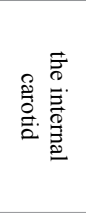 } & \multicolumn{3}{|c|}{ the backmost of turning } & 3.829214 & 6.397832 & -3.19416 & 8.111584 \\
\hline & \multicolumn{3}{|c|}{ the foremost of turning } & 3.698031 & 10.80676 & -3.15536 & 11.8498 \\
\hline & \multicolumn{3}{|c|}{ the innermost of turning } & 1.369086 & 8.740381 & -3.20355 & 9.409112 \\
\hline & \multicolumn{3}{|c|}{ the outermost of turning } & 5.98296 & 8.250021 & -3.20025 & 10.68177 \\
\hline & \multicolumn{3}{|c|}{ the nearest point } & 0.896478 & 7.386872 & -0.73228 & $\begin{array}{c}7.477017 \\
(7.480636)\end{array}$ \\
\hline \multirow{14}{*}{ 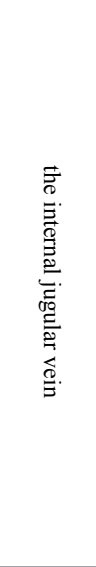 } & \multicolumn{3}{|c|}{ the superior midpoint at the juncture of sigmoid sinus } & 0.834106 & -3.52514 & -7.42257 & 8.259352 \\
\hline & \multicolumn{3}{|c|}{ the backmost at the juncture of sigmoid sinus } & -0.32397 & -4.16201 & -9.99769 & 10.83426 \\
\hline & \multicolumn{3}{|c|}{ the foremost at the juncture of sigmoid sinus } & 3.154102 & -1.89381 & -9.74179 & 10.41333 \\
\hline & \multicolumn{3}{|c|}{ the uppermost at the juncture of sigmoid sinus } & 2.631649 & -3.08045 & -6.67203 & 7.805814 \\
\hline & \multicolumn{3}{|c|}{ the lowest at the juncture of sigmoid sinus } & 1.176184 & -3.56193 & -10.9731 & 11.59654 \\
\hline & \multicolumn{3}{|c|}{ the outermost at the juncture of sigmoid sinus } & 3.903221 & -2.94646 & -7.95202 & 9.33549 \\
\hline & \multicolumn{3}{|c|}{ the innermost at the juncture of sigmoid sinus } & -0.58825 & -4.1433 & -9.30429 & 10.2021 \\
\hline & \multicolumn{3}{|c|}{ at the juncture of inferior petrosal sinus } & -1.07523 & 0.363311 & -8.43979 & 8.51576 \\
\hline & \multicolumn{3}{|c|}{ the highest point of jugular fossa } & 0.935054 & -1.36589 & -6.17055 & 6.388714 \\
\hline & & ermost at the juncture of $c$ & & -0.43695 & -3.64986 & -11.7273 & 12.28991 \\
\hline & & termostat the juncture of $c$ & & 1.635853 & -2.58388 & -11.4744 & 11.87494 \\
\hline & the & ermost at the juncture of & & 0.077814 & -4.03588 & -11.1648 & 11.87212 \\
\hline & & west at the juncture of co & & 0.239981 & -2.49206 & -12.2656 & 12.5185 \\
\hline & & the nearest point & & 0.810381 & -1.09384 & -6.19603 & $\begin{array}{c}6.343815 \\
(6.345607)\end{array}$ \\
\hline & & the lowest & & 9.076244 & -7.0245 & -12.8102 & 17.19951 \\
\hline 管离 & & the nearest point & & 2.185261 & -3.13426 & -6.79354 & $\begin{array}{c}7.794302 \\
(7.793747)\end{array}$ \\
\hline & & & $\mathbf{X} \mathbf{a}$ & & $Y$ axis & $\mathrm{Z}$ axis & distance \\
\hline cochleari & rocess & the center & 10.63 & & 5.650994 & 1.514502 & 12.13552 \\
\hline pyramic & nence & the apex & 11.83 & & 3.527317 & -0.39108 & 12.36036 \\
\hline & & the anterior midpoint & 8.946 & & 4.189309 & 1.35602 & 9.97126 \\
\hline 1 & & the posterior midpoint & 9.498 & & 3.453821 & 1.071247 & 10.1637 \\
\hline oval & & the superior midpoint & 9.422 & & 3.849192 & 1.451082 & 10.2809 \\
\hline & & the inferior midpoint & 9.141 & & 3.930295 & 0.92442 & 9.993487 \\
\hline rounc & ow & the center & 9.063 & & 3.099325 & -0.01995 & 9.578757 \\
\hline
\end{tabular}

* Values inside the brackets of the tables representing the distances measured in the original coordinate and then converted to length.

Creation of coordinate system using the external lower lip of the internal acoustic pore as its origin

\section{Selection of location marks}

The external lower lip of the internal acoustic pore has often been regarded as the initiation point of measuring the distance from the internal acoustic pore to relevant structures as a result of being easy to recognize [2]. Therefore we choose the external lower lip of the internal acoustic pore as coordinate origin to measure the $3 \mathrm{D}$ coordinate of the internal structures.

The significance of the creation of coordinate system using the external lower lip of the internal acoustic pore as its origin

Gupta et al. [3] determined that $5 \mathrm{~mm}$ of the posterior border of the internal acoustic meatus could be safely stripped after measuring on the head of dead body that the distance from the inferior border of the right internal acoustic pore to the highest point of jugular fossa was $7.94 \pm 1.47 \mathrm{~mm}$ while the vertical distance between them being $2.77 \pm$ $1.63 \mathrm{~mm}$, the shortest distance from the posterior lip of the internal acoustic pore to the external aperture of aqueduct of vestibule $9.86 \pm$ $1.54 \mathrm{~mm}$. However, the safe depth of stripping could not be determined due to failed measurement of the distance between the internal acoustic pore and the posterior semicircular canal. The distance measured in our study from the external lower lip of the internal acoustic pore was $6.39 \mathrm{~mm}$ to the highest point of jugular fossa and $7.25 \mathrm{~mm}$ to the external aperture of aqueduct of vestibule, which conforms to the results of the above-mentioned study. The shortest distance to the posterior semicircular canal was $7.56 \mathrm{~mm}$ and it could be concluded from the $3 \mathrm{D}$ coordinate that the distance from the external lower lip of the internal acoustic pore was $0.94 \mathrm{~mm}$ outward, $1.37 \mathrm{~mm}$ backwards and $6.17 \mathrm{~mm}$ downward to the highest point of jugular fossa, $6.48 \mathrm{~mm}$ outward, $2.78 \mathrm{~mm}$ backwards and $1.70 \mathrm{~mm}$ downward to the external lower port of the vestibular aqueduct and $7.38 \mathrm{~mm}$ outward, $0.86 \mathrm{~mm}$ forward, and $1.42 \mathrm{~mm}$ upward to the nearest point from the posterior semicircular canal. The safe range of stripping during operation could be determined with more accuracy according to 3D coordinate system.

Day et al. [2] measured 8 values of distance between the lower lateral lip of the internal acoustic pore and relevant structures in the posterior surface of the petrous bone from 32 samples of temporal bone, among which the length of the internal acoustic meatus stripped was $5.9 \pm 1.4 \mathrm{~mm}$ and the distances from the external lower lip of the internal acoustic pore to common crus and to vestibule were $10.4 \pm$ 
$2.1 \mathrm{~mm}, 9.5 \pm 1.9 \mathrm{~mm}$ respectively. It is shown in our study that the distances from the external lower lip of the internal acoustic pore to the superior and inferior border, posterior and anterior transverse ridge of the fundus of internal acoustic meatus were $8.03 \mathrm{~mm}, 5.03 \mathrm{~mm}$, $6.96 \mathrm{~mm}$ and $7.19 \mathrm{~mm}$ respectively, while the shortest distances to the inner side of common crus and to vestibule were $7.66 \mathrm{~mm}$ and 7.49 $\mathrm{mm}$. All of these are close to that obtained by Diaz, suggesting that our experimental data are accurate and reliable. As the fundus of internal acoustic meatus represents a range rather than a point, it is more precise for us to measure the distances from the external lower lip of the internal acoustic pore to the superior, inferior, anterior and posterior borders of it. It extends $7.46 \mathrm{~mm}$ outward, $1.25 \mathrm{~mm}$ forward, $1.20 \mathrm{~mm}$ upward from the external lower lip of the internal acoustic pore to the innermost side of common crus and $7.29 \mathrm{~mm}, 1.43 \mathrm{~mm}, 0.91 \mathrm{~mm}$ to the closest point of the vestibule, which enables us to understand its structure and position with more accuracy in terms of space. Shimizu et al. [4] chose 27 adult dry temporal bones to simulate the opening of the posterior wall of internal acoustic meatus through retro sigmoid approach and conducted relevant measurements. It was $10.6 \mathrm{~mm}$ from the posterior border of the internal acoustic pore to the superior border of the endolymphatic sac, $7.51 \mathrm{~mm}$ to the vestibular aqueduct, and 7.27 $\mathrm{mm}$ to the inner side of common crus, laying anatomical foundation for avoiding damage to structures like the endolymphatic sac when tumor occurs in the internal acoustic meatus. The results of our experiment are also very close to that.

We have made systematic measurements of the coordinates of the external lower lip of the internal acoustic pore to the intersection point between each semicircular canal and the vestibule, the interior and exterior of topmost semicircular canal as well as the closest and farthest distances to the semicircular canal, thus basically determining the coordinate about the general appearance of the semicircular canal. Coordinates related to the cochlea and vestibule serve data basis for not impairing them during operation. It enables us to determine the labyrinth, horizontal and vertical sections of the facial nerve according to the coordinates about the starting point of the facial canal, geniculate ganglion and the plane of pyramidal eminence, contributing to the quick positioning of the facial nerve and the design of operative approach. The coordinates about the starting point, turning and the external aperture of the vestibular aqueduct provide data and information for not causing damage to the endolymphatic duct and sac. It requires us to be very familiar with the three dimensional relations and coordinate positions of the internal carotid canal and internal jugular vein inside the petrous bone as well as sigmoid sinus, and inferior petrosal sinus so as to avoid massive haemorrhage during operation. The coordinates about the cochleariform process, pyramidal eminence, oval window and round window help us to understand the spatial relation between the middle ear and the inner ear. Due to lack of time, only 3D coordinate of one petrous bone on the right side has been established in this study, so further measurements of coordinate values from large samples are needed in the future in order to discover rules, identify the range of coordinate values and thus provide better guidance for clinicians to carry out operation.

\section{Ethical standards}

The authors assert that all procedures contributing to this work comply with the ethical standards of The Ethics Committee of Tianjin Medical University on human experimentation and with the Helsinki Declaration of 1975, as revised in 2008.

\section{A bullet point summary}

The three-dimensional model of the petrous bone has clearly displayed the detailed surface and internal structures of the petrous bone and their spatial relationships. We first established a three dimensional physical coordinate system with the external lower lip of the internal acoustic pore as origin to conduct an exact positioning research into the internal composition of the petrous bone.

\section{References}

1. Wei XF, Zhang XY, Yuan W, Li YS (2015) Accuracy of computer-aided geometric three-dimensional reconstruction of the human petrous bone based on serial unstained celloidin sections. Experimental and therapeutic medicine 9: 1113-1118. [Crossref]

2. Day JD, Kellogg JX, Fukushima T, Giannotta SL (1994) Microsurgical anatomy of the inner surface of the petrous bone: neuroradiological and morphometric analysis as an adjunct to the retrosigmoid transmeatal approach. Neurosurgery 34: 1003-1008. [Crossref]

3. Gupta T, Gupta SK (2009) Anatomical delineation of a safety zone for drilling the internal acoustic meatus during surgery for vestibular schwanomma by retrosigmoid suboccipital approach. Clinical Anatomy 22: 794-799. [Crossref]

4. Shimizu S, Tanaka R, Oka H, Fujii K (2006) Risk of damage to the endolymphatic sac and duct during removal of the posterior meatal wall: an anatomic study. Neurosurgery 59: ONS435-ONS439. [Crossref]

Copyright: (C2016 Wei XF. This is an open-access article distributed under the terms of the Creative Commons Attribution License, which permits unrestricted use, distribution, and reproduction in any medium, provided the original author and source are credited. 